\title{
Cosmic Rays
}

\section{The cosmic ray spectrum between $1 \mathrm{GeV}$ and $10^{4} \mathrm{GeV}$ and the radio emission from supernova remnants}

\author{
Peter L. Biermann ${ }^{1}$ and Richard G. Strom ${ }^{2}$ \\ 1 Max Planck Institut für Radioastronomie, Auf dem Hügel 69, D-5300 Bonn 1, Germany \\ 2 Netherlands Foundation for Research in Astronomy, Radiosterrenwacht, P.O.Box 2, NL-7990 AA Dwingeloo, The Netherlands
}

Accepted for publication, March 1993

\begin{abstract}
.
Based on a conjecture about the diffusion tensor of relativistic particles perpendicular to the magnetic field at a shock, and considering particle drifts, we develop a theory to account for the cosmic ray spectrum between $1 \mathrm{GeV}$ and $10^{4} \mathrm{GeV}$. The essential assumption is that the mean free path perpendicular to the magnetic field is independent of energy and has the scale of the thickness of the shocked layer. We then use the basic concept that the cosmic ray particles are accelerated in a supernova shock that travels through the interstellar medium. Radio observations, with additional supporting evidence from optical and X-ray data, suggest that small additional and transient bowshocks in locally free expansion contribute to the injection, while it is the overall shock which accounts for injection as well as the main acceleration of energetic particles, and we obtain a reasonable picture of the physical process with such a concept. Physically important ingredients besides the presence of a strong shock are diffusion, drifts, convection, adiabatic cooling, the injection history, and the topology of the magnetic field, here assumed for simplicity to be homogeneous in the interstellar medium. The result is a spectrum, which for strong shocks in a gas with adiabatic index $5 / 3$ yields a spectrum of $E^{-2.42}$. Interstellar turbulence with a Kolmogorov spectrum then leads by leakage from the galactic disk to a spectrum which is $E^{-2.75}$, as observed in cosmic rays, and as deduced from radio observations of the nonthermal emission from our Galaxy as well as that of all other well-observed galaxies. We argue that the ratio of cosmic ray electrons to protons is determined by the amount of expansion which takes place from the cessation of electron injection to the break-up of the shell by cooling instabilities. Since the highest particle energy reached derives from geometrical arguments, it depends on the charge of the nucleus and so higher $Z$ elements are predicted to reach higher energies.
\end{abstract}

Key words: acceleration of particles - cosmic rays - diffusion - supernovae - supernova remnants - shock waves

Send offprint requests to: Peter L. Biermann

\section{Introduction}

The origin of cosmic rays is still not completely understood. There are a few well-accepted arguments:

a) The cosmic rays below about $310^{4} \mathrm{GeV}$ are due to the explosions of stars into the normal interstellar medium (Lagage \& Cesarsky 1983);

b) The cosmic rays from near $10^{3} \mathrm{GeV}$ up to the knee, at $510^{6} \mathrm{GeV}$, are very likely due to explosions of massive stars into their former stellar winds (Völk \& Biermann 1988). The consequences of this concept have been checked by calculating the cosmic ray abundances and comparing them with observations (Silberberg et al. 1990); the comparison suggests that up to the highest energy where abundances are known, this concept successfully explains the data. It is especially interesting that no mixing from the supernova ejecta is required to account for the known cosmic ray abundances, Wolf Rayet winds and other strong stellar winds around evolved stars as sources are all that is needed at present;

c) For the energies beyond the knee there is no consensus; Jokipii \& Morfill (1987) argue that a galactic wind termination shock might be able to provide these particles, while Protheroe \& Szabo (1992) propose an extragalactic origin, although in either case the matching of the flux at the knee from two different source populations remains somewhat problematic;

d) For the cosmic rays beyond the ankle, at about $310^{9} \mathrm{GeV}$ an extragalactic origin is required because of the extremely large gyroradii of such particles.

In the first paper of this series (Biermann 1993, hereafter paper CR I) we have proposed that a major effect to consider is the transport coefficient in a geometry where the magnetic field is perpendicular to the shock normal, commonly referred to as a perpendicular shock, and where the shock is spherical. Then we investigate the propagation of a shock wave in either a stellar wind or into a homogeneous medium. In such a case particle drifts at the shock and in the upstream and downstream regions are important (see, e.g., Jokipii 1987).

Observations can be a guide here: The explosions into the interstellar medium are explosions where the magnetic field is nearly perpendicular to the shock direction over most of $4 \pi$ steradians. Radio polarization observations of supernova remnants clearly indicate what the typical local structure of these 
shocked plasmas is. The observational evidence (Milne 1971, Downs \& Thompson 1972, Reynolds \& Gilmore 1986, Milne 1987, Dickel et al. 1988b) has been summarized by Dickel et al. (1991) in the statement that all shell-type supernova remnants less than 1000 years old show dominant radial structure in their magnetic fields near their boundaries. There are several possible ways to explain this: Rayleigh-Taylor instabilities between ejected and swept-up material can lead to locally radial differential motion and so produce a locally radial magnetic field (Gull 1973). It could be due to strong radial velocity gradients of various clumps of ejecta, or result from clouds being overrun that now evaporate and cool the surrounding material (all of which are mentioned by Chevalier [1977]).

It may seem paradoxical that we will be considering perpendicular shocks where the observations show a parallel magnetic field, but it must be remembered that the observed field is a superposition of the configurations of all emitting regions in the telescope beam. The degree of polarization in all of the objects referred to above is low (typically $10 \%$ from a perfectly uniform field (generally $65 \%$ being both parallel and perpendicular components present. In young shells the former dominate, presumably reflecting the importance of the radial motions involved. (Although not relevant to the polarization, it might be noted that the parallel components will cancel on average, while the perpendicular components do not since they derive from the preexisting magnetic field structure.)

The radial magnetic field configuration is also found in a few shell remnants somewhat older than $1000 \mathrm{yr}$, such as RCW 86 (SN 185) and Pup A (with a kinematic age of some 3700 yr, Winkler et al. 1988), showing that radial motion in some form persists beyond the youthful stage. In most "mature" remnants, however, the field is tangential, or complex (Milne 1987). While a radially-directed magnetic field provides the most compelling evidence for large-scale differential motions parallel to the shock direction, there are other indications as well. It is generally believed that the oxygen-rich fast moving knots (fmk's) in Cas A are ejecta from the progenitor (Kamper \& Van den Bergh 1976). Oxygen-rich knots have also been seen in Pup A (Winkler et al. 1988) and the similarly aged G 292.0+1.8 (Braun et al. 1986), and may also be present in a number of extragalactic SNRs (Winkler \& Kirshner 1985). Radially-directed radio structures have been mapped in Cas A, and their motion has also been determined (Braun et al. 1987). Recent Rosat observations of the older (10 000 yr) Vela SNR show similar features in the X-ray emission which have apparently penetrated the main SNR shell (plenary talk by J. Trümper presented at the Texas/Pascos '92 Symposium, December 1992) and are believed to result from fmk-like ejecta. (Their very soft X-ray spectra would make similar features difficult to detect in more distant remnants.)

The important conclusion for us here is that there appear to be strong radial differential motions in perpendicular shocks which provide the possibility that particles get convected parallel to the shock direction. We assume this to be a diffusive process, and note that others have also pointed out that diffusion may be the key to understanding shock acceleration (e.g. Falle 1990). Our task here will be to derive a natural velocity and a natural length scale, which can be combined to yield a diffusion coefficient. A classical prescription is the method of Prandtl (1925): In Prandtl's argument an analogy to kinetic gas theory is used to derive a diffusion coefficient from a natural scale and a natural velocity of the system. Despite many weaknesses of this generalization Prandtl's theory has held up remarkably well in many areas of science far beyond the original intent. We will use a similar prescription here.

Consider the structure of a layer shocked by a supernova explosion into a homogeneous medium in the case that the adiabatic index of the gas is $5 / 3$ and the shock is strong. Then there is an inherent scale in the system, namely the thickness of the shocked layer, in the spherical case for a strong shock limit $r / 12$. There is also a natural velocity scale, namely the velocity difference of the flow with respect to the two sides of the shock. Both are the smallest dominant scale in velocity and in length; we will use the assumption that the smallest dominant scale is the relevant scale again to derive diffusion coefficients and other scalings.

In paper CR I we introduced the basic theory on the basis of a transport coefficient as described above, and in paper CR II (Biermann \& Cassinelli 1993) we tested it with the observations of nonthermal radio emission from accelerated particles in shocks traversing stellar winds, while in paper CR IV (Stanev et al. 1993) we checked the spectrum and chemical composition against air shower data in the particle energy range from $10^{4} \mathrm{GeV}$ to $310^{9} \mathrm{GeV}$. The interpretation of the radio observations of stars before and after they explode led us to the concept that there is a critical shock velocity of order $10^{9} \mathrm{~cm} \mathrm{~s}^{-1}$ above which electrons get injected into the acceleration process in a fashion similar to the nuclei, and below which this injection is severely restricted, an effect of several orders of magnitude. One possible choice for this critical velocity is that at which the post-shock thermal electrons reach relativistic velocities, or, in equipartition, the whistler velocity becomes relativistic.

Biermann (1992), Rachen (1992), Rachen \& Biermann (1992, 1993: paper UHE CR I) have proposed that the ultrahigh energy particles arise from hot spots in nearby radio galaxies; this hypothesis leads to a successful and nearly parameterfree explanation (Rachen 1992) of the intensity, chemical composition and spectrum of these particles (see Rachen et al. 1993, paper UHE CR II) with the important proviso that the mean free path in intergalactic space should be not much smaller than the characteristic distances between the sources and us, and may be similar to the scale of the large-scale bubbles in the universe. These results independently confirm the existence of a steep spectrum component of heavy nuclei, identified with galactic sources in paper CR IV.

The observations of the cosmic rays themselves, and of the nonthermal radio emission from our Galaxy as well as from all other well-observed galaxies strongly suggest that in all carefully studied galactic environments, the cosmic rays have a universal spectrum of very nearly $E^{-8 / 3}$ below the knee at $510^{6} \mathrm{GeV}$ (below about $10 \mathrm{GeV}$ for the energetic electrons); direct air shower experiments show the spectrum beyond the knee to be well-approximated by $E^{-3}$. This overall spectrum is clearly influenced by propagation effects, since particles at different energies have different probabilities of escaping from the disk of the galaxy. It appears to be a reasonable hypothesis to approximate the interstellar turbulence spectrum by a Kolmogorov law, which leads to an interstellar diffusion coefficient proportional to $E^{1 / 3}$. Such an energy dependence then requires a source spectrum of Cosmic Rays of approximately $E^{-7 / 3}$ below the knee, and approximately $E^{-8 / 3}$ above the knee.

In this paper we propose to derive such a spectrum for particle energies below about $10^{4} \mathrm{GeV}$. The basic hypothesis 
is, again, that we consider explosions into a homogeneous interstellar medium. We will use the standard Sedov solution to the expansion of such an explosion into the interstellar medium (e.g. Cox 1972).

\section{Definition of the task}

We consider the acceleration of particles in a shock that propagates into a homogeneous magnetic interstellar medium. The acceleration of particles is governed by the standard theory (Parker 1965), which includes the effects of first, time change, then radial diffusion, latitude diffusion, radial drift, latitude drift, then compression and finally sources (see paper CR I). The orientation which we use is such that the symmetry axis is parallel to the prevailing magnetic field, and we assume that to first order the magnetic field structure is unidirectional and homogeneous in the undisturbed interstellar medium. We take the cosine $\mu$ of the colatitude as our coordinate along $\theta$. The interstellar magnetic field is known to contain both systematic ordered components and a random component (Beck 1986) of similar strength; hence we will demonstrate below (Sect. 8) that the simplification of using a uniform field does not introduce any unnecessary restrictions. The components of the diffusion tensor of interest here are the radial diffusion term $\kappa_{r r}$ and the latitude diffusion term $\kappa_{\theta \theta}$.

The Sedov similarity solution for an explosion into a homogeneous medium can be written as a function of shock radius $r$ and shock speed $U_{1}$; as a function of time $t_{4}$, in units of $10^{4}$ years; explosion energy $E_{51}$, in units of $10^{51}$ ergs; and density of the interstellar medium $n_{\mathrm{o}}$ in units of $\mathrm{cm}^{-3}$. This gives (Cox 1972)

$r=13.7 t_{4}^{2 / 5}\left(E_{51} / n_{\mathrm{o}}\right)^{1 / 5} \mathrm{pc}$

and

$$
\begin{aligned}
U_{1} / c & =1.810^{-3} t_{4}^{-3 / 5}\left(E_{51} / n_{\mathrm{o}}\right)^{1 / 5} \\
& =0.090(r / \mathrm{pc})^{-3 / 2}\left(E_{51} / n_{\mathrm{o}}\right)^{1 / 2} .
\end{aligned}
$$

Among the modifications which can be made to Eqs. (1) and (2) with practical application to SNRs, it is possible to account for (radial) density gradients in both the ambient medium and the ejecta. Chevalier (1982) suggests, for example, that a modification which may be appropriate for Tycho would result in $r \propto t^{4 / 7}$ instead of the $2 / 5$ power-law in Eq. (1). Kinematic investigations of Tycho (Strom et al. 1982), Kepler (Dickel et al. 1988a) and SN 1006 (Long et al. 1988) show that while there are deviations from the ideal $2 / 5$ power-law, they are not substantial, and it is moreover unknown what their precise cause is. We shall consequently adopt the Sedov solution for the sake of simplicity and because it seems to be a fair representation of reality.

Our boundary conditions are the usual: We inject particles at some low energy which is assumed to be independent of all relevant properties of the problem, i.e. not dependent on radial distance, magnetic field strength or latitude. Downstream we assume the flow to take particles out of the system with the normal probability $4 U_{2} / c$ (see, e.g., Drury 1983), where $U_{2}$ is the downstream velocity relative to the shock.

We propose to derive the essential properties of the particle distribution function by analytical means, using heuristic arguments. The key will be the form of the diffusion tensor, especially the radial component $\kappa_{r r}$.

\section{Diffusion perpendicular to the average magnetic field}

Our basic conjecture (see CR I), then, is that the convective random walk of energetic particles perpendicular to the magnetic field can be described by a diffusive process due to fast convective motions with a downstream diffusion coefficient $\kappa_{r r, 2}$ which is given by the thickness of the shocked layer together with the velocity difference across the shock, and is thus independent of energy:

$\kappa_{r r, 2}=\frac{1}{9} \frac{U_{2}}{U_{1}} r\left(U_{1}-U_{2}\right)$

Here we have assumed that in the combination of transport coefficients perpendicular and parallel to the magnetic field (see Sect. 8) the perpendicular convective transport dominates. As is obvious from Eqs. (51) and (52) below, this is a very good approximation for nearly all angles over the $4 \pi$, since the parallel diffusion coefficient is linear with $E$, and the perpendicular convective transport is independent of particle energy. The exact limiting condition is $\left(1-\mu^{2}\right) / \mu^{2} \gg E / E_{\max }$, which defines the very small solid angle over which the parallel diffusion is stronger.

The upstream diffusion coefficient is then obtained (again, see paper CR I) by using the same velocity scale and the downstream length scale multiplied by the density ratio, which gives $r / 3$ for a strong shock. Again, the gyroradius of the same maximum particle energy as in the shocked layer gives the same scale. Since the magnetic field is lower by a factor of $U_{2} / U_{1}$ upstream, the gyroradius of the maximum particle energy upstream is also $r / 3$. Hence the upstream diffusion coefficient is

$\kappa_{r r, 1}=\frac{1}{9} r\left(U_{1}-U_{2}\right)$

It immediately follows that

$\frac{\kappa_{r r, 1}}{r U_{1}}=\frac{\kappa_{r r, 2}}{r U_{2}}=\frac{1}{9}\left(1-\frac{U_{2}}{U_{1}}\right)$

For these diffusion coefficients, it also follows that the residence times (Drury 1983) on both sides of the shock are equal and are

$\frac{4 \kappa_{r r, 1}}{U_{1} c}=\frac{4 \kappa_{r r, 2}}{U_{2} c}=\frac{4}{9} \frac{r}{c}\left(1-\frac{U_{2}}{U_{1}}\right)$.

Adiabatic losses then cannot limit the energy reached by any particle since they run directly with the acceleration time, both being independent of energy, and so the limiting size of the shocked layer limits the energy that can be reached to that where the gyroradius just equals the thickness of the shocked layer. This then leads to a potential maximum energy of

$E_{\max }=\frac{1}{3} \frac{U_{2}}{U_{1}} Z \operatorname{er} B_{2}=\frac{1}{3} Z \operatorname{er} B_{1}$

where $Z e$ is the particle charge and $B_{1,2}$ is the magnetic field strength on the two sides of the shock. This means, once again, that the energy reached corresponds to the maximum gyroradius the system will allow. It also means that we push the diffusive picture right up to its limit where the diffusive scale becomes equal to the mean free path and the gyroradius of the most energetic particles. We will derive a more restrictive condition below (Sect. 8). 


\section{Particle Drifts}

Consider particles which are either upstream of the shock, or downstream; as long as the gyrocenter is upstream we will consider the particle to be there, and similarly downstream.

In general, the energy gain of the particles will be governed primarily by their adiabatic motion in the electric and magnetic fields. The expression for the energy gain is well known and given in, e.g., Northrop (1963, equation 1.79), for an isotropic angular distribution where one term comes from the drifts and a second from the induced electric field (see paper CR I). We explicitly work in the shock frame, separate the two terms and consider the drift term first. The second term is accounted for further below, in Sect. 5 .

The $\theta$-drift can be understood as arising from the asymmetric component of the diffusion tensor, the $\theta r$-component. The natural scales there are the gyroradius and the speed of light, and so we note that for (Forman et al. 1974)

$\kappa_{\theta r}=\frac{1}{3} r_{\mathrm{g}} c$

the exact limiting form derived from ensemble averaging, we obtain the drift velocity by taking the proper covariant divergence (Jokipii et al. 1977); this is not simply (spherical coordinates) the $r$-derivative of $\kappa_{\theta r}$. The general drift velocity is given by (see, e.g., Jokipii 1987)

$V_{\mathrm{d}, \theta}=c \frac{E}{3 Z e} \operatorname{curl}_{\theta} \frac{\mathbf{B}}{B^{2}}$.

The $\theta$-drift velocity is thus zero upstream and only finite downstream due to curvature:

$V_{\mathrm{d}, \theta}=\frac{c}{3} \frac{r_{\mathrm{g}}}{r}$

where $r_{\mathrm{g}}$ is now taken to be positive (see above).

It must be remembered that there is a lot of convective turbulence which increases the curvature: The characteristic scale of the turbulence is $r / 12$ for strong shocks, and thus the curvature is $12 / r$ maximum. Taking half the maximum as average we obtain then for the curvature a factor of $6 / r$ which is six times the curvature without any turbulence; this increases the curvature term by a factor of six thus changing its contribution from $1 / 3$ to 2 in the numerical factor in Eq. (10). Hence the total drift velocity is

$V_{\mathrm{d}, \theta}=\frac{1}{2}\left(\frac{U_{1}}{U_{2}}\right) \frac{c r_{\mathrm{g}}}{r}$,

now written for arbitrary shock strength. It is easily verified that the factor in front of Eq. (11) is two for strong shocks where $U_{1} / U_{2}=4$. With $\Delta E_{1}=0$ we have then downstream

$\frac{\Delta E_{2}}{E}=\frac{2}{9} \frac{U_{1}}{c}\left(1-\frac{U_{2}}{U_{1}}\right)$

which is the total energy gain from drifts.

Let us consider then one full cycle of a particle remaining near the shock and cycling back and forth from upstream to downstream and back. The energy gain just due to the Lorentz transformations in one cycle can then be written as

$$
\left(\frac{\Delta E}{E}\right)_{\mathrm{LT}}=\frac{4}{3} \frac{U_{1}}{c}\left(1-\frac{U_{2}}{U_{1}}\right)
$$

Adding the energy gain due to drifts, Eq. (12), we obtain

$\frac{\Delta E}{E}=\frac{4}{3} \frac{U_{1}}{c}\left(1-\frac{U_{2}}{U_{1}}\right) x$

where

$x=1+\frac{1}{6}$.

\section{Source expansion and particle injection history}

5.1. Expansion and injection history, shell

Consider how long it takes a particle to reach a certain energy:

$\frac{d t}{d E}=\left[8 \frac{\kappa_{r r, 1}}{U_{1} c}\right]\left[\frac{4}{3} \frac{U_{1}}{c}\left(1-\frac{U_{2}}{U_{1}}\right) x E\right]^{-1}$.

Here we have used $\kappa_{r r, 1} / U_{1}=\kappa_{r r, 2} / U_{2}$. Since we have, from the Sedov solution [Eqs. (1) and (2)], $r=(5 / 2) U_{1} t$, this leads to,

$\frac{d t}{t}=\frac{d E}{E} \frac{3 U_{1}}{U_{1}-U_{2}} \frac{2}{x} \frac{\kappa_{r r, 1}}{r U_{1}} \frac{5}{2}$

and so to a dependence of

$t(E)=t_{\mathrm{o}}\left(\frac{E}{E_{\mathrm{o}}}\right)^{\beta}$

with

$\beta=\frac{3 U_{1}}{U_{1}-U_{2}} \frac{2}{x} \frac{\kappa_{r r, 1}}{r U_{1}} \frac{5}{2}$.

Particles that were injected some time $t$ ago were injected at a different rate, say, proportional to $r^{b}$. Also, in a $d$-dimensional space, particles have $r^{d}$ more space available to them than when they were injected, and so we have a correction factor which is

$\left(\frac{E}{E_{\mathrm{o}}}\right)^{-\frac{2}{5}(b+d) \beta}$.

The combined effect [Eqs. (19) and (20)] is a spectral change by

$\frac{3 U_{1}}{U_{1}-U_{2}} \frac{2}{x}(b+d) \frac{\kappa_{r r, 1}}{r U_{1}}$.

We note that the factor $2 / 5$ from the Sedov expansion drops out again. For a detailed argument on the sign conventions used here, see paper CR I. This expression can be compared with a limiting expansion derived by Drury (1983; eq. 3.58), who also allowed for a velocity field. Drury's expression agrees with the more generally derived expression given here. This also describes then the adiabatic losses, due to the general expansion of the shock layer. Hence the total spectral difference, as compared with the plane parallel case, is given by

$\frac{3 U_{1}}{U_{1}-U_{2}}\left[\frac{U_{2}}{U_{1}}\left(\frac{1}{x}-1\right)+\frac{2}{x}(b+d) \frac{\kappa_{r r, 1}}{r U_{1}}\right]$.

If this expression is positive the spectral index is steeper.

Furthermore, we have to discuss the effect of the expansion of the material in the shell, in this context already introduced by Drury (1983). This expansion is only that beyond the linear expansion of the overall flow already taken into account. We define the velocity for the flow field $U(\xi r)$ as a function of radius $\xi r$ interior to the shock radius $r$ by 
$U(\xi r)=V(\xi) \xi U_{1}$

We thus have, e.g., $V(1)=V^{\prime}(1)=3 / 4$. This velocity field can be approximated by

$V(\xi)=3 \frac{\xi^{8}+1}{3 \xi^{8}+5}$.

As shown by Drury (1983), this velocity field introduces a steepening by

$\frac{3 U_{1}}{U_{1}-U_{2}} \frac{\kappa_{r r, 2}}{r U_{2}} \frac{V^{\prime}(1)}{V(1)}$

neglecting drifts and in the limit of very small diffusion coefficient. However, in our context where we consider a finite shell, we have to use a non-local approximation. To do this we simply equate the expansion of the hot interior to the shell with the postshock speed of sound, which is given by

$c_{2}=\left(\frac{2 \gamma}{\gamma-1}\right)^{1 / 2} U_{2}$,

where the numerical factor is $\sqrt{5}=2.23608 \ldots$ for an adiabatic constant of $\gamma=5 / 3$. The postshock gas expands at the shock in the observer's frame at $U_{1}-U_{2}$, which is $3 U_{2}$ for a strong shock. The linear expansion itself already provides $\left(U_{1}-U_{2}\right) / 12$, which is $U_{2} / 4$. Hence the extra expansion over linear is $2.75 U_{2}-2.23608 U_{2}$. Using then finite differences instead of derivatives, we have

$\frac{V^{\prime}(1)}{V(1)} \rightarrow \frac{2.75 U_{2}-\sqrt{5} U_{2}}{3 U_{2}} \frac{23}{24} 12=1.970$

instead of unity as in Drury's case, which is $1.97007 \ldots$ times the term from the local flow field $V(\xi)$. Here the factor $23 / 24$ comes from averaging the postshock radius from 1 to $1-1 / 12$, and thus describing the average of $\xi / \Delta \xi$. Hence we have an extra term from this postshock adiabatic loss due to the shell expansion of

$\frac{3 U_{1}}{U_{1}-U_{2}} \frac{1}{x} \frac{\kappa_{r r, 2}}{r U_{2}} \frac{V^{\prime}(1)}{V(1)} 1.970$,

which is then slightly less than double the effect which Drury discusses. For a strong shock then this particular effect adds 0.563 to the spectral index.

For strong shocks in stellar winds, for which the natural length scale is $r / 4$, the linear part of the expansion is three times higher and so the remaining difference to the postshock speed of sound is only $2.25-\sqrt{5}$, which gives an effect of only $210^{-3}$ in the spectral index, justifying a posteriori our neglect of this effect in papers CR I and CR II.

The exponent $b$ [Eq. (20)] describes the injection as a power of the radius; in a Sedov solution the injection, assumed to be proportional to $\rho U_{1}^{2}$, is given by

$\rho U_{1}^{2} \sim r^{-3}$.

Hence we have $b=-3$, so that $b+d=0$. Thus we have for a pure Sedov solution, using the velocity field term introduced above, a spectral difference to the plane parallel case of 0.444 and thus an injection spectrum of
$E^{-2.420}$

However, we note that this spectrum is valid for electrons obviously only if they are injected into the acceleration process at all, i.e. if the shock speed is above the critical velocity for electron injection. Adding the term for diffusive losses from the Galaxy the final observable spectrum expected is

$E^{-2.753}$

very close to what is observed, both in Galactic cosmic rays (protons and nuclei), as well as through the nonthermal radio emission of other galaxies (Golla 1989, electrons).

Clearly, there is uncertainty in our method for treating the non-linear flow field; the various ways of correcting the term Eq. (25) all lead to numerical correction factors very close to 2.0; above we have taken 1.97. Further non-linear corrections, such as also averaging the non-linear flow field itself, lead to spectral indices within 0.04 of the value given in Eq. (30).

\subsection{Expansion and injection history, fast moving knots}

Here we have to note that the radio images of Cas A (Anderson et al. 1991) suggest that there are many small additional bowshocks which in fact may do some of the particle acceleration (see especially Braun et al. 1987). They are associated with compact clumps related to the fmk/ejecta (which, in fact, they may well be), and have travelled outwards essentially undecelerated until their encounter with the high density material associated with the shock. Since the main shock has undergone significant deceleration (Tuffs 1986, Braun et al. 1987), the clumps, expelled with a range of radial speeds, are now able to overtake it, brightening and hence becoming prominent as they do. Each clump is the apex of a bowshock which is radially oriented, and as it passes the shock front it becomes a fresh start into the interstellar medium and so can be presumed to be in free expansion for as long as it survives, until it ultimately merges back into the overall general flow. The observations suggest that these bowshocks, initiated by small blobs of denser material, move with velocity constant in time, but different for different blobs; when such blobs happen to move faster than the present average velocity of the shell, they must have penetrated it in the past, but when their velocity is just the current value of $r / t=(5 / 2) U_{1}$, then they have just caught up with, and hence penetrate, the shell now.

The drift velocity is then given by a consideration of free expansion into the homogeneous medium, and we approximate this free expansion here as a spherical shell of which only a segment is realized. Writing for the blob radius $r_{\mathrm{b}}$ and its velocity $U_{\mathrm{b}}$ we then obtain for the drift, again using the argument about convective scales [e.g. Eq. (11)],

$V_{\mathrm{d}, \theta}=\frac{1}{2} \frac{U_{\mathrm{b} 1}}{U_{\mathrm{b} 2}} \frac{r_{\mathrm{g}}}{r_{\mathrm{b}}} c$.

The downstream residence time is then

$\tau_{\mathrm{res}, 2}=\frac{1}{3} \frac{r_{\mathrm{b}}}{3} \frac{U_{\mathrm{b} 2}}{U_{\mathrm{b} 1}}\left(U_{\mathrm{b} 1}-U_{\mathrm{b} 2}\right) \frac{4}{U_{\mathrm{b} 2} c}$.

The energy gain due to drifts is then given by

$\frac{\Delta E}{E}=\frac{2}{9} \frac{U_{\mathrm{b} 1}}{c}\left(1-\frac{U_{\mathrm{b} 2}}{U_{\mathrm{b} 1}}\right)$. 
Now the argument above suggests that $U_{\mathrm{b} 1}=(5 / 2) U_{1}$. Using then the plausible assumption that $U_{\mathrm{b} 1} / U_{\mathrm{b} 2}=U_{1} / U_{2}$ we obtain

$\frac{\Delta E}{E}=\frac{5}{9} \frac{U_{1}}{c}\left(1-\frac{U_{2}}{U_{1}}\right)$.

Adding this now to the Lorentz transformation energy gain (but also with the increased shock speed) we obtain for the parameter $x$ again the value $x=7 / 6$ [Eq. (15)]. In order to calculate the resulting spectrum we have to find now the effective diffusion coefficient and express it in terms of the shell parameters, such as shell thickness of the overall explosion, for strong shocks $r / 12$. For free expansion the parameters $b$ and $d$ are $b+d=3$, and the expansion is linear so that the velocity field is $V^{\prime}(\xi)=0$. Hence we have to fix the residence time now in order to determine the spectrum. Now the total scale of the blob-induced convective turbulence is, upstream plus downstream, $(5 / 4) r_{\mathrm{b}} / 3$. This entire scale will grow until at some point it reaches the overall shell scale $r / 12$; then by virtue of the switch-over in scales the overall shell scale will begin to dominate and the blob expansion will cease to be important. This means that

$r_{\mathrm{b}}=\frac{r}{5}$

and also that

$r_{\mathrm{b}} U_{\mathrm{b} 1}=\frac{1}{2} r U_{1}$.

Correspondingly we have to insert $\kappa_{r r, 1} /\left(r U_{1}\right)=1 / 24$ and so obtain for the spectral difference from the plane parallel case 0.715 , which gives an injection spectrum of

$E^{-2.715}$

nearly $1 / 3$ steeper than the spectrum for the overall shock acceleration, Eq. (30), suggesting that these smaller shocks provide just some injection for the larger shocks. However, the smaller shocks may not only run into a geometric limit, they are likely to run into an energetic limit even earlier (i.e. no longer have sufficient energy to be in free expansion locally), and so cease to be important before reaching the limit derived above.

As can be seen from the expression for the spectral index, it depends on the blob radius, and thus on expansion time. The spectrum steepens with blob radius $r_{\mathrm{b}}$, which is proportional to expansion time for as long as the blob is in local free expansion. The spectrum implied is $E^{-2.114}$ at $0.3 r_{\mathrm{b}}$, then $E^{-2.715}$ at exactly $r_{\mathrm{b}}$, to steepen to $E^{-4.429}$ at $3 r_{\mathrm{b}}$. Since energetic particles experience both the effect of the small blobs as well as that of the overall shock, the overall spectral index for the blobs is expected to be slightly steeper than the average (nonblob) spectral index of the shell, consistent with observations. We thus surmise on energetic grounds that these blobs break up below the critical blob size given above, and note that their radio emission does in fact fade rapidly as they pass the shock front (Braun et al. 1987), which is consistent with the breakup requirement. This means that the blobs are likely to change over to a local Sedov expansion before reaching the critical blob size; this then would lead to a cessation of electron injection as soon as the the shock velocity drops below the critical velocity.
The spectrum derived is the one injected by small spherical blobs in local free expansion. This spectrum has more power proportional to the square of the shock velocity, since the shock velocity $U_{\mathrm{b} 1}$ is $5 / 2$ higher than the overall shell shock velocity $U_{1}$, and also the effective area is, of course, approximately half a sphere, giving another factor of two over the area of a planar circle on the large shell. Hence the multitude of these little blobs puts about one order of magnitude more power per area of the large shell into the injection than the spherical shell shock directly. We conclude that the small blobs driven by the fast moving knots may contribute substantially to the injection of particles, just as surmised by Braun et al. (1987). (Parenthetically, it should be noted that the possible role of fmk's in particle acceleration was first investigated by Scott \& Chevalier [1975] who, however, assumed a second-order Fermi process in the turbulent fmk wakes.)

We add that these small bow shocks are not the only objects that should have time-variable radio emission; radio observations suggest that there are also many nearly stationary magnetic substructures, with likely a magnetic field up to a factor 5 above that given by the shock-enhanced interstellar field of about $20 \mu \mathrm{G}$. Such substructures can arise when the overall expansion overruns preexisting features in the interstellar medium, for which a straightforward analogy with the solar photosphere would suggest that magnetic substructures of much stronger magnetic fields might be quite common.

There is one immediate check: Braun et al. (1987) and Anderson et al. (1991) emphasize that the large bow shock features with the steepest radio spectral index have the strongest time variation of their flux density. In our simple concept this can be interpreted as the consequence of two effects: First, with the size of the blob or bow shock the region emitting scales as the third power of the radius of the blob and so steadily increases. Second, with the steeper spectrum the weight of the particle spectrum is shifted to low particle energies and so the radio emission is increased. For going from a radio spectral index of, e.g., -0.6 to -0.7 the combined effect is about a factor of 4 , equally caused by both effects.

The spectrum derived is very close to the observed galactic cosmic ray spectrum at low energies and we propose that this is the mechanism to generate it.

\section{The time evolution of the radio spectral index}

Since the shock velocity is steadily decreasing with time for a Sedov expansion, we have to consider the effect of particle acceleration through Alfvén wave scattering, commonly referred to as second order Fermi acceleration. The importance of this mode of acceleration has been appreciated for some time (e.g., Scott \& Chevalier 1975, Chevalier et al. 1976).

The energy gain through this effect can be included in our derivation of $\Delta E / E$ :

$$
\left(\frac{\Delta E}{E}\right)_{2 \mathrm{~F}}=\left(\frac{\Delta E}{E}\right)_{\mathrm{LT}}\left(\frac{v_{\mathrm{A}}}{U_{1}}\right)^{2} \text { const } \sim\left(\frac{\Delta E}{E}\right)_{\mathrm{LT}} r^{3} .
$$

This translates into a time-dependent value of $x$ :

$x=1.167+\left(r / r_{\mathrm{s}}\right)^{3}$.

This in turn translates into a spectral index for the particle distribution which gets flatter with time and expansion radius: 
$1+\frac{1.657}{1.167+\left(r / r_{\mathrm{s}}\right)^{3}}$

The radio spectral index of the nonthermal synchrotron emission then has a radius-dependence accordingly of

$\frac{0.842}{1.167+\left(r / r_{\mathrm{s}}\right)^{3}}$

This leads to a gradual flattening of the spectral index with $r$. The quantitative behaviour of this simple approximation is in agreement with the data shown for the few supernova remnants for which we have adequate distances or know their individual time evolutions (Anderson et al. 1991, Fig. 1).

We note that these effects depend on the ambient density $n_{\mathrm{o}}$ such that for a low density environment the Alfvén velocity is high and hence second order Fermi acceleration may then become important. However, we have to emphasize that second order Fermi acceleration depends on the average strength of the Alfvénic wave field across the entire shell region, and since that is unknown, we can derive the temporal change of the spectral index only to within an unknown factor, which might be rather small. Thus we see two ways to explain the trend for overall spectral index to flatten with diameter (Berkhuijsen 1986, Anderson et al. 1991):

First, there is the possibility that for larger and thus older remnants the spectral index may in fact be due to a combination of various components that may have formed over the long time scale available: 1) HII regions with their rather flat radio spectrum from free-free emission, 2) a pulsar driven supernova remnant which also has a flatter radio spectrum, and 3) an underlying synchrotron spectrum of a shell-type supernova remnant as discussed in this paper. Furthermore, the supershells often found in other galaxies require the energy input of many stellar winds and/or supernovae and might be an analogy to the extremely large supernova remnants detected in our Galaxy, and not just attributable to a single event.

Second, one could argue that only a few remnants survive to the age where second order Fermi acceleration becomes important, and so these remnants are not significant for the typical injection spectrum for the release of cosmic rays into the interstellar medium. The distribution of supernova remnants is given by a $r^{+1 / 2}$ power law in equal logarithmic bins of observed radius, for any given environmental density $n_{\mathrm{o}}$. The adiabatic expansion covers a range of only $8.1 n_{\mathrm{o}}^{-0.12}$ from free expansion to cooling and break up, and thus this range is rather insensitive to the density. However, the breakup radius depends as $n_{\mathrm{o}}^{-5 / 11}$ on density and so the typical range of at least a factor of 100 in the interstellar medium density (see, e.g., Fig. 12 in Berkhuijsen 1986) likely to be experienced by exploding stars will shift the distribution around by an extra factor of ten, totally mixing the diagram (see, e.g., Fig. 11 in Berkhuijsen 1986). Thus, it is close to impossible to disentangle these various effects all of which lead to selection effects in any observational survey. We suspect, however, that it is this second alternative which is more reasonable in our Galaxy.

\subsection{The time evolution of the radio emission}

Calculating now the radio emission contribution from the shell of $r U_{2} /\left(3 U_{1}\right)$, we note that the ram pressure is proportional to $U_{1}^{2}$, which in the Sedov solution [Eq. (2)] is proportional to $r^{-3}$. The total radio emission from the shell is then constant with time if the efficiency of injecting electrons, $\eta$, is a constant. The luminosity is then given by

$L_{\nu}=5.410^{23} B_{-5.3}^{1.710} \eta_{-1} E_{51} \nu_{9.0}{ }^{-0.710} \mathrm{erg} \mathrm{s}^{-1} \mathrm{~Hz}^{-1}$

and so does not depend on the interstellar medium density; since the adiabatic expansion is a condition of constant energy, all such dependences drop out. We have used here as reference 0.1 for $\eta, 5 \mu \mathrm{G}$ for the unperturbed interstellar magnetic field strength, and $1 \mathrm{GHz}$ for the emission frequency observed. We note that the emission here is coming from a shell of thickness $r / 12$; additional emission further inside might arise if there is a reverse shock resulting from the transition of free expansion to adiabatic expansion. Evidence for reverse-shock heating of ejecta may exist in X-ray images of Tycho (Seward et al. 1983 ), and has also been construed from X-ray spectroscopy of SN 1006 (Hamilton et al. 1986a) and Tycho (Hamilton et al. $1986 \mathrm{~b}$.

Furthermore, there is observational evidence that the synchrotron emission systematically samples the higher magnetic field substructures, by a factor of up to 5 and so we can expect the synchrotron emission to be higher by factors up to 15.7 , leading to an implied radio luminosity of up to $8.510^{24} \mathrm{erg} \mathrm{s}^{-1} \mathrm{~Hz}^{-1}$, everything else being equal. This luminosity is in agreement with the upper limit of the distribution of observed luminosities, suggesting that in their initial phases the efficiency of putting kinetic energy into an energetic electron population is indeed of order 0.1 .

However, when we derive the normalization factor for the particle spectrum to use in the approximation that the total energy in all relativistic particles is $\eta$ times the flow energy, we have implicitly used a constant particle spectrum. After having shown how we might understand the early time evolution of the spectral index, we now have to calculate how this influences the normalization; since with an increasingly flatter spectrum the energy in the particle population shifts to higher values, there are increasingly fewer particles at lower energy, the very particles responsible for radio emission. However, this effect is only strong for relativistic particles and is counterbalanced by the non-relativistic sector of momentum space, so that the entire effect is only a factor of order 2 for a change from a synchrotron spectral index of -0.7 to -0.8 . Therefore, the radio emission is expected to decrease slowly with time. As soon as electron injection ceases, then simple aging will decrease the electron spectrum rapidly and Shklovsky's argument holds (Shklovsky $1968, \S 7$ ), demonstrating how the electron population decreases simply due to overall adiabatic losses.

\section{The injection into the interstellar medium}

Since the particle spectrum flattens with time and the observed cosmic ray spectrum corresponds to the early spectral index of Sedov expansion explosions, there is an apparent discrepancy. This discrepancy can be resolved by noting that a Sedov type expansion breaks up when the shell becomes unstable to cooling; the observations and our interpretation then suggest that most supernova remnants break up and release their energetic particle population into the interstellar medium long before second order Fermi acceleration becomes relevant. The Vela supernova remnant may be an example of a shell that has been breaking up for some time. Spectroscopy has shown that the motions in Vela are very chaotic (Jenkins et al. 1984), 
with large departures from simple radial motion, which may be evidence for this.

The evolution of a supernova remnant (see, e.g., for the basic physical concepts employed Cox 1972) is then divided into four phases:

First, there is free expansion until the interstellar medium overrun by the explosion shock is of about the same mass as the mass ejected. This happens at a radius of

$R_{\mathrm{e}}=1.92\left(\frac{M_{\mathrm{ej}}}{M_{\odot}}\right)^{1 / 3} n_{\mathrm{o}}^{-1 / 3} \mathrm{pc}$,

where $M_{\mathrm{ej}}$ is the ejected stellar mass and $n_{\mathrm{o}}$ is again the ambient density in $\mathrm{cm}^{-3}$. Numerical simulations demonstrate that the expansion is noticeably slowed down long before this critical radius is reached.

Second, we have a Sedov phase with adiabatic expansion and steady fresh electron injection. The observations of radio emitting stars suggest that there is a critical velocity for a shock, below which electron injection is severely restricted. The critical speed is of order

$U_{1, \mathrm{crit}}=c\left(m_{\mathrm{e}} / m_{\mathrm{p}}\right)^{1 / 2} \simeq 7000 \mathrm{~km} \mathrm{~s}^{-1}$,

as suggested by the radio luminosities of OB and WR stars on the one hand, and radio supernovae on the other (CR II); this comparison suggests just the approximate velocity, not the actual number, but the particular velocity given here is consistent with the stellar data. It is highly unlikely that the same critical velocity would be relevant in the interstellar medium, and so we only use the concept that there is such a critical velocity and adopt as reference $1000 \mathrm{~km} \mathrm{~s}^{-1}\left(=U_{\text {crit, }-2.5}\right.$, in units of $c$ ) on the grounds that this would correspond to roughly the same Alfvénic Mach number as the supernova shocks in stellar winds as argued in papers CR I and CR II without any physical justification at this stage. We plan to test such a concept with further stellar examples of nonthermal radio emission. In our picture the injection is done by both the bow shocks around the fast moving knots and the overall shock. Hence the critical radius is given by

$R_{\text {crit }}=9.0\left(E_{51} / n_{\mathrm{o}}\right)^{1 / 3} U_{\text {crit },-2.5}^{-2 / 3} \mathrm{pc}$.

Third, when the expansion velocity has decreased below the critical speed, the electron injection ceases and we have further adiabatic expansion, but with the electron population only modified by adiabatic losses. Adiabatic losses of a relativistic particle decrease its energy by the ratio of the initial and final radius scales.

And finally, fourth, we have the break-up phase, which occurs because the cooling layer is thermally unstable (McCray et al. 1975, Chevalier and Imamura 1982, Smith 1989) leading finally to a break-up of the supernova shell. The cooling radius is (Cox 1972)

$R_{\text {cool }}=15.6 E_{51}^{3 / 11}\left(10^{22} L\right)^{-2 / 11} n_{\mathrm{o}}^{-5 / 11} \mathrm{pc}$,

where $L$ is the cooling coefficient; the cooling curve has been compared with recent detailed calculations by Schmutzler \& Tscharnuter (1993) and is still a very good approximation at the temperature range of interest here. During this time the energy of any individual relativistic electron decreases by adiabatic expansion as the ratio of the radii from the time when injection ceases to the time of break up. Thus the energy density of the electron population (from conservation of the adiabatic moment only and disregarding spatial dilution, see below) decreases by the factor $(p=2.420)$

$$
\left(\frac{R_{\text {cool }}}{R_{\text {crit }}}\right)^{p-1}=2.18 E_{51}^{-0.09} n_{\mathrm{o}}^{-0.17}\left(10^{22} L\right)^{-0.26} U_{\text {crit },-2.5}^{0.95},
$$

showing a very weak dependence on the environmental density $n_{\mathrm{o}}$. The dependence on the critical velocity is very nearly linear. The simple spatial dilution of the energy density is the same for both protons and electrons and drops out in the ratio, as long as we are in the Sedov phase (see Shklovsky [1968], eq. 7.27). For a tenuous interstellar medium of $\mathrm{H}$-atom number density about $0.01 \mathrm{~cm}^{-3}$, the factor is increased to nearly 4.8 .

This intermediate switch from electron injection with steady acceleration to a simple adiabatic loss regime will determine the net scaling of the power of the electron population to that of the proton population in the cosmic rays. The observations suggest that from $1 \mathrm{GeV}$ the electron number is only about 0.01 (Wiebel 1992) of the protons. From this observed ratio the energy density ratio integrated over the entire relativistic part of the particle spectrum, protons relative to electrons, for the injection spectral index of -2.42 , is given by 4.3. Assuming then that the ratio of electrons to protons is not influenced by propagation effects, this suggests an expansion of a factor in radius of order 3 between the time when electron injection ceases and the time when cooling breaks up the shell of the supernova remnant; here we assume that electrons and protons originally have comparable energy densities of their relativistic particle populations.

Interestingly, this is very close to the ratio implied above for normal parameters. Below, where we argue about the maximum particle energy that can be attained, we see that the tenuous part of the interstellar medium is likely to be the phase responsible for the injection. This is then fully consistent with our adopted particular value for the critical velocity of 1000 $\mathrm{km} \mathrm{s}^{-1}$ and suggests that we may have identified the physical reason for the observed electron/proton ratio.

\section{The maximum energy of particles}

The maximum energy particles can possibly reach is given in Sect. 3, and depends linearly on the magnetic field. Near the symmetry axis we have diffusion parallel to the magnetic field, and, given the strong turbulence induced by the shock we take there the Bohm limit for the upstream diffusion coefficient:

$\kappa_{\|}=\frac{1}{3} \frac{E}{Z e B_{1}} c$.

At the equator we use our radial upstream diffusion coefficient derived earlier, here for a strong shock, of

$\kappa_{\perp}=\frac{r U_{1}}{12}$.

Combining Eqs. (49) and (50) for the two upstream diffusion coefficients (Jokipii 1987) to obtain an acceleration time scale and setting that equal to the upstream flow time scale of $r /\left(3 U_{1}\right)$ then yields a condition on the maximum particle energy that can be reached. This condition is 
$\frac{r}{3 U_{1}}=\frac{4}{3} \frac{E}{Z e B_{1}} \frac{c}{U_{1}^{2}} \mu^{2}+\frac{r}{3 U_{1}}\left(1-\mu^{2}\right)$.

This leads to

$E_{\max }=\frac{1}{4} Z e B_{1} r \frac{U_{1}}{c}$.

A more detailed consideration of the strength of the magnetic field as a function of latitude does not change this result. In addition, since here the actual coordinate $\mu$ does not appear in the expression, it is obvious that this condition holds locally over any area of the spherical shock with a locally homogeneous magnetic field. Therefore, the overall inhomogeneity of the magnetic field does not change this result.

Using normal interstellar magnetic fields of $5 \mu \mathrm{G}$, and a standard Sedov explosion with an arbitrary radius then yields a maximum particle energy of

$E_{\max }($ protons $)=1.010^{5}(r / \mathrm{pc})^{-1 / 2}\left(E_{51} / n_{\mathrm{o}}\right)^{1 / 2} \mathrm{GeV}$

and

$E_{\max }($ iron $)=3.010^{6}(r / \mathrm{pc})^{-1 / 2}\left(E_{51} / n_{\mathrm{o}}\right)^{1 / 2} \mathrm{GeV}$.

At breakup, when we suggest the actual mixing of the particle population into the interstellar medium occurs, these maximum energies are

$E_{\max }($ protons $)=2.610^{4} F_{\mathrm{CR}} \mathrm{GeV}$,

and

$E_{\max }($ iron $)=7.410^{5} F_{\mathrm{CR}} \mathrm{GeV}$.

where we use

$F_{\mathrm{CR}}=E_{51}^{0.364}\left(10^{22} L\right)^{0.091} n_{\mathrm{o}}^{-0.273}$.

The protons ought to dominate due to the normal abundances in the interstellar medium, and higher energies for protons are clearly reached in the tenuous hot part of the interstellar gas where the densities are of order $0.01 \mathrm{~cm}^{-3}$, and so maximum proton energies are possible to about $1.010^{5} \mathrm{GeV}$. These numbers are similar to earlier estimates of the maximum particle energy in a Sedov expansion phase of a supernova. In paper CR IV we obtain from a fit to the air shower data an estimate for this number of $1.210^{5} \mathrm{GeV}$, fully consistent with these arguments, and suggesting that the cosmic ray injection is most effective in the tenuous part of the interstellar medium, an argument which has been made before on quite different grounds.

\section{Summary}

In this third paper of a series on the process of cosmic ray acceleration we have again used a basic conjecture (Sect. 3) on the diffusion of particles in a shock perpendicular to the magnetic field. In a similar vein we have introduced a number of heuristic arguments which require testing against observations. We have already tested and further explored the consequences of this concept in another paper (CR II) on the nonthermal radio emission of Wolf-Rayet stars and demonstrate that our concept can produce the proper radio spectral indices, luminosities and temporal behaviour. In a further communication we have tested in detail the predictions of this model as regards the chemical abundances of cosmic rays (CR IV). What we have shown in papers CR I, II, IV and here, is that the entire cosmic ray spectrum from $1 \mathrm{GeV}$ out to $310^{9} \mathrm{GeV}$, including the knee energy and the chemical abundances, can be understood with the same physical ingredients already well-tested in the solar wind shock, namely a strong shock, diffusion, drifts, convection, adiabatic cooling, and injection history.

In this paper in particular, we have used the concept introduced in paper CR II of a critical velocity for electron injection to fashion an argument for the observed electron/proton ratio in cosmic rays. We have derived an estimate for the upper particle energy that can be reached in such explosions, of order $10^{5} \mathrm{GeV}$, close to what had been argued previously and what is required by the air shower data (paper CR IV). We have also derived the proper cosmic ray spectrum, consistent with direct measurements as well as with radio observations. It obviously follows that the abundances in such cosmic rays ought to be close to those of the interstellar medium itself, as is observed.

Acknowledgements. The essential part of this work was carried out during a five-month sabbatical in 1991 of PLB at Steward Observatory at the University of Arizona, Tucson. PLB wishes to thank Steward Observatory, its director, Dr. P.A. Strittmatter, and all the local colleagues for their generous hospitality during this time and during many other visits. PLB also wishes to thank Drs. E. Berkhuijsen, D.C. Ellison, T.K. Gaisser, J.R. Jokipii, K. Mannheim, H. Meyer, R. Protheroe, S.P. Reynolds, M.M. Shapiro, T. Stanev and R. Tuffs for extensive discussions of cosmic ray, supernova and stellar physics. High energy physics with PLB is supported by grants DFG Bi 191/6,7, 9 (Deutsche Forschungsgemeinschaft), BMFT grant (DARA FKZ 50 OR 9202) and NATO travel grant (to T. Stanev and PLB) CRG 910072. The research of RGS is supported by the Netherlands Organisation for Scientific Research (NWO).

\section{References}

Anderson, M., Rudnick, L., Leppik, P., Perley, R., Braun, R. 1991, ApJ, 373, 146

Beck, R. 1986, IEEE Transactions on Plasma Science, PS-14, 740

Berkhuijsen, E.M. 1986, A\&A, 166, 257

Biermann, P.L. 1992, in "Frontiers in Astrophysics", Eds. R. Silberberg, G. Fazio, M. Rees, Cambridge Univ. Press (in press since 1990, release scheduled for June 1993)

Biermann, P.L. 1993, A\&A, in press (CR I)

Biermann, P.L., Cassinelli, J.P. 1993, A\&A submitted (CR II)

Braun, R., Goss, W.M., Caswell, J.L., Roger, R.S. 1986, A\&A, 162,259

Braun, R., Gull, S.F., Perley, R.A. 1987, Nat, 327, 395

Chevalier, R.A. 1975, ApJ, 200, 698

Chevalier, R.A. 1977, ARA\&A, 15, 175

Chevalier, R.A. 1982, ApJ, 258, 790

Chevalier, R.A., Imamura, J.N. 1982, ApJ, 261, 543

Chevalier, R.A., Robertson, J.W., Scott, J.S. 1976, ApJ, 207, 450

Cox, D.P. 1972, ApJ, 178, 159

Dickel, J.R., Sault, R., Arendt, R.G., Matsui, Y., Korista, K.T. 1988a, ApJ, 330, 254

Dickel, J.R., Sault, R., Arendt, R.G., Matsui, Y., Korista, K.T. 1988b, ApJ, 333, 259 
Dickel, J.R., Breugel, W.J.M. van, Strom, R.G. 1991, AJ, 101, 2151

Downs, G.J., Thompson, A.R. 1972, AJ, 72, 120

Drury, L.O'C. 1983, Rep. Prog. Phys., 46, 973

Falle, S.A.E.G. 1990, in "Neutron Stars and their Birth Events", Ed. W. Kundt, Kluwer, Dordrecht, p. 303

Forman, M.A., Jokipii, J.R., Owens, A.J. 1974, ApJ, 192, 535

Golla, G. 1989, M. Sc. Thesis, University of Bonn

Gull, S.F. 1973, MNRAS, 161, 47

Hamilton, A.J.S., Sarazin, C.L., Szymkowiak, A.E. 1986a, ApJ, 300, 698

Hamilton, A.J.S., Sarazin, C.L., Szymkowiak, A.E. 1986b, ApJ, 300, 713

Jenkins, E.B., Wallerstein, G., Silk, J. 1984, ApJ, 278, 649

Jokipii, J.R. 1987, ApJ, 313, 842

Jokipii, J.R., Levy, E.H., Hubbard, W.B. 1977, ApJ, 213, 861

Jokipii, J.R., Morfill, G. 1987, ApJ, 312, 170

Kamper, K., Van den Bergh, S. 1976, ApJS, 32, 351

Lagage, P., Cesarsky, C. 1983, A\&A, 118, 223

Long, K.S., Blair, W.P., Van den Bergh, S. 1988, ApJ, 333, 749

McCray, R., Stein, R.F., Kafatos, M. 1975, ApJ, 196, 565

Milne, D.K. 1971, Aust. J. Phys., 24, 757

Milne, D.K. 1987, Aust. J. Phys., 40, 771

Northrop, Theodore G. 1963, "The Adiabatic Motion of Charged Particles", Interscience Publishers, New York

Parker, E.N. 1965, Planet. Space Sci., 13, 9

Prandtl, L. 1925, Zeitschrift angew. Math. und Mech., 5, 136

Protheroe, R.J., Szabo, A.P. 1992, Phys. Rev. Letters, 69, 2885

Rachen, J. 1992, M. Sc. Thesis, University of Bonn

Rachen, J., Biermann, P.L. 1992, in "Particle acceleration in cosmic plasmas", Eds. G.P. Zank, T.K. Gaisser, AIP Conf. No.264, p. 393

Rachen, J., Biermann, P.L. 1993, A\&A, in press (UHE CR I)

Rachen, J., Stanev, T., Biermann, P.L. 1993, A\&A, in press (UHE CR II)

Reynolds, S.P., Gilmore, D.M. 1986, AJ, 92, 1138

Schmutzler, T., Tscharnuter, W. 1993, A\&A, in press

Scott, J.S., Chevalier, R.A. 1975, ApJ, 197, L5

Seward, F., Gorenstein, P., Tucker, W. 1983, ApJ, 266, 287

Shklovsky, I.S. 1968, "Supernovae", Wiley-Interscience, London

Silberberg, R., Tsao, C.H., Shapiro, M.M., Biermann, P.L. 1990, ApJ, 363, 265

Smith, M.D. 1989, MNRAS, 238, 235

Stanev, T., Biermann, P.L., Gaisser, T.K. 1993, A\&A, in press (CR IV)

Strom, R.G., Goss, W.M., Shaver, P.A. 1982, MNRAS, 200, 473

Tuffs, R.J. 1986, MNRAS, 219, 13

Völk, H.J., Biermann, P.L. 1988, ApJ, 333, L65

Wiebel, B. 1992, unpublished HEGRA-Note, University of Wuppertal

Winkler, P.F., Kirshner, R.P. 1985, ApJ, 299, 981

Winkler, P.F., Tuttle, J.H., Kirshner, R.P., Irwin, M.J. 1988, in "Supernova Remnants and the Interstellar Medium", Eds. R.S. Roger, T.L. Landecker, Cambridge University Press, Cambridge, p. 65

This article was processed by the author using Springer-Verlag $\operatorname{IAT}_{\mathrm{E}} \mathrm{X}$ A\&A style file 1990. 